\title{
Research on manipulator grasping method based on vision
}

\author{
Lei Yang ${ }^{1, *}$, Shiliang $\mathrm{Wu}^{1}$, Zhenlong $\mathrm{Lv}^{1}$, and Feng $\mathrm{Lu}^{1}$ \\ ${ }^{1}$ School of Mechatronic Engineering and Automation, Shanghai University, China
}

Keywords: Static grab, Vision, Direct linear method, Trajectory function.

\begin{abstract}
Aiming at the problem that the manipulator cannot grasp the object accurately when the robot changes the position and pose of the object in the process of static grasping, a set of manipulator grasping method research based on vision is proposed. Firstly, a set of camera detection and robot grasping system model is built. Secondly, each coordinate system is created for the grasping system, and the transformation relation between each coordinate system, matrix model and the quantity that needs to be calibrated are introduced in detail. Thirdly, the trajectory function from the image coordinate system to the coordinates of the manipulator is obtained through the direct linear method calibration experiment. Finally, the experimental platform for camera detection and manipulator grasping objects based on xavis was built, and the grasping success rate of the experimental platform was tested. The experimental results show that the grasping error rate of the experimental platform is within the control range. Therefore, the manipulator grasping method based on vision is of reference significance for engineering applications.
\end{abstract}

\section{Introduction}

Under the background of increasing labor costs, difficulty in recruitment, increasing demand for diversified production and stable development of automation technology, more and more industrial robots are involved in industrial production activities, among which solving the problem of product positioning and grasping is an important problem that industrial robots will inevitably encounter in practical application [1-2].In the past, the most common solution was to fix the position and posture of the product, and then control the industrial robot to grab the product through manual teaching in advance and action reproduction in the later stage [3]. When the actual position of the product deviates from the teaching position, the traditional way is difficult to meet the requirements. The emergence and development of machine vision technology provide new solutions for the positioning of industrial robots [4].

\footnotetext{
* Corresponding author: yanglei130@shu.edu.cn
} 
Liu Zheng-qiong introduced how to realize the recognition of parts larger than the camera field of view based on local feature matching [5]. Ni He-peng. introduced image de-repetition algorithm and acceleration and deceleration control algorithm in the process of robot tracking. Zheng hua. mainly studied CSARA robot operational model and methods of extracting and processing visual image features [6].Chen Zhi-wei. introduced the image processing, control algorithm and control program in the system of visual guidance parallel robot [7].

The above literatures focus on the research of visual image extraction and processing methods, or the dynamics control of the robot itself, but do not involve the coordinate system pose data transformation and calibration methods among vision, conveyor belt and industrial robot. In this paper,the conveyor belt, intelligent camera and industrial robot as research objects, proposes a complete set of object pose data calculation methods based on camera detection[8]. Through the transformation formula of coordinate matrix created, only the visual detection data need to be read, and the pose data of the product coordinate system in the robot world coordinate system can be calculated automatically[9].

\section{Construction of camera detection and robot grasping system platform}

Firstly, the visual robot is fixed on the robot operating platform through the chassis, and then a single visual robot workbench is assembled through the connector. The two visual robots are distributed on both sides of the conveyor belt. The physical position layout of mechanical arm, camera and assembly base in the robot grasping system platform is shown in figure 1. Select one of the arms to complete the grab task. The specific placement size is shown in table 1 , and the robot static tracking and grasping system is shown in figure 2.
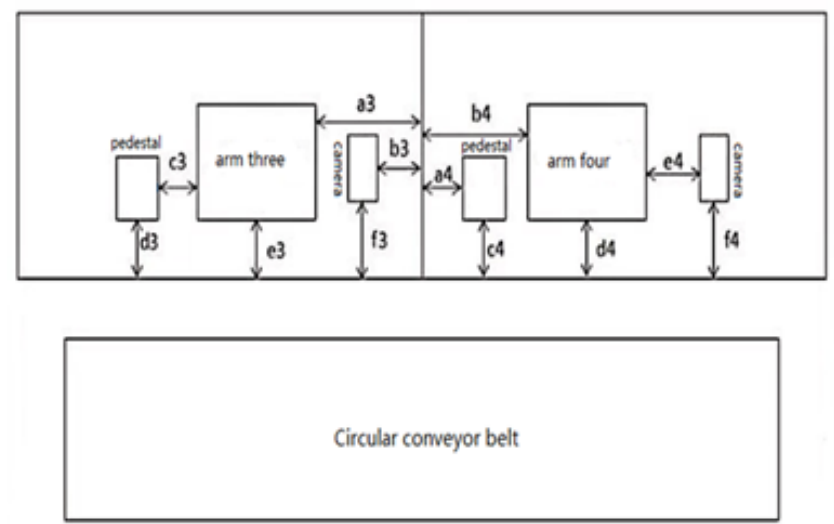

Fig. 1. The grasping system platform.

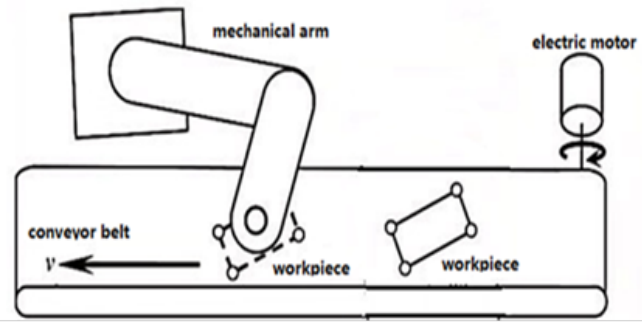


Fig. 2. The tracking and grasping system.

Table 1. Size table for each object.

\begin{tabular}{ccccccc}
\hline Serial number & $\mathrm{a} 3(\mathrm{~cm})$ & $\mathrm{b} 3(\mathrm{~cm})$ & $\mathrm{c} 3(\mathrm{~cm})$ & $\mathrm{d} 3(\mathrm{~cm})$ & $\mathrm{e} 3(\mathrm{~cm})$ & $\mathrm{f} 3(\mathrm{~cm})$ \\
\hline distance & 19 & 2 & 13.4 & 6.9 & 4 & 11.6 \\
Serial number & $\mathrm{a} 4$ & $\mathrm{~b} 4$ & $\mathrm{c} 4$ & $\mathrm{~d} 4$ & $\mathrm{e} 4$ & $\mathrm{f}$ \\
distance & 0.5 & 19 & 6.9 & 4 & 11.7 & 11.6 \\
\hline
\end{tabular}

\section{Kinematics analysis of manipulator}

\subsection{Analysis of the relationship between the creation and transformation of coordinate system}

The objects controlled in this paper are all eye-in-hand systems[10]. The following is an eye-in-hand robot as an example to illustrate the relationship between the coordinate systems of the system. Let $\{\mathrm{W}\}$ represent the base coordinate system of the robot, $\{\mathrm{E}\}$ represent the coordinate system of the robot end-effector, $\{C\}$ represent the camera coordinate system, $\{I\}$ represent the image plane coordinate system, and $\{\mathrm{O}\}$ represent the target object coordinate system[11]. The position relationship between the coordinate systems is shown in figure 3 .

\subsection{D-H parametric method}

In this paper, D-H parameter method is used to model the mechanical arm[12]. Firstly, the coordinate system of each link is established separately, the pose relation of adjacent link is obtained by using homogeneous transformation matrix, and the pose relation of the coordinate system of end-effector relative to the base coordinate system is obtained by transformation in turn, and the kinematics model of the manipulator is established. The parameters of the D-H of figure is shown in figure 4.The position and attitude of one coordinate system relative to another can be obtained by coordinate transformation. This relationship can be described by four homogeneous transformation $T(i)$ matrices.

$$
\begin{gathered}
{ }^{i-1} T_{i}=\operatorname{Rot}\left(x_{i}, \alpha_{i-1}\right) \operatorname{Trans}\left(x_{i}, a_{i-1}\right) \operatorname{Trans}\left(z_{i}, d_{i}\right) \operatorname{Rot}\left(z_{i}, q_{i}\right) \\
=\left[\begin{array}{cccc}
\cos q_{i} & -\sin q_{i} \cos \alpha_{i-1} & \sin q_{i} \sin \alpha_{i-1} & a_{i-1} \cos q_{i} \\
\sin q_{i} & \cos q_{i} \cos \alpha_{i-1} & -\cos q_{i} \sin \alpha_{i-1} & a_{i-1} \sin q_{i} \\
0 & \sin \alpha_{i-1} & \cos \alpha_{i-1} & d_{i} \\
0 & 0 & 0 & 1
\end{array}\right]
\end{gathered}
$$

Equation(1) is the homogeneous matrix of the transformation of robot connecting rod. The transformation between the coordinate systems of connecting rod with any degree of complexity can be described by taking the product of the appropriate number of the matrix. In the control of the manipulator, it is usually necessary to obtain the spatial pose relationship between the tool coordinate system $\{\mathrm{T}\}$ and the universal coordinate system $\{\mathrm{S}\}$. The transformation matrix of tool coordinate system and universal coordinate system can be finally obtained by measuring the position and pose relationship ${ }^{i-1} T_{i}$ with the base coordinate system. 


$$
{ }_{T}^{S} T_{T}={ }_{B}^{S} T_{T}^{B} T
$$

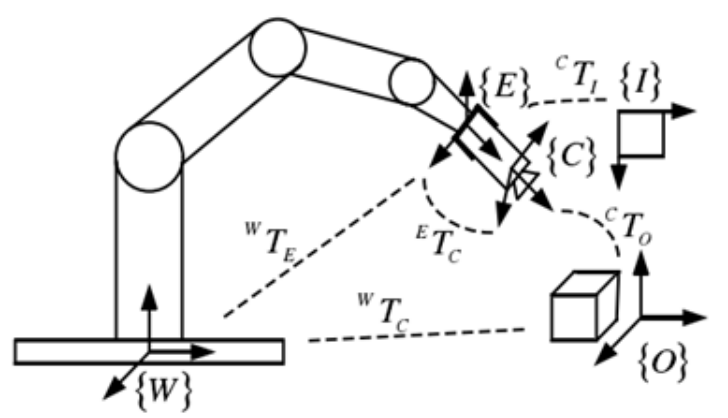

Fig. 3. The coordinate relation of robot grasping system.

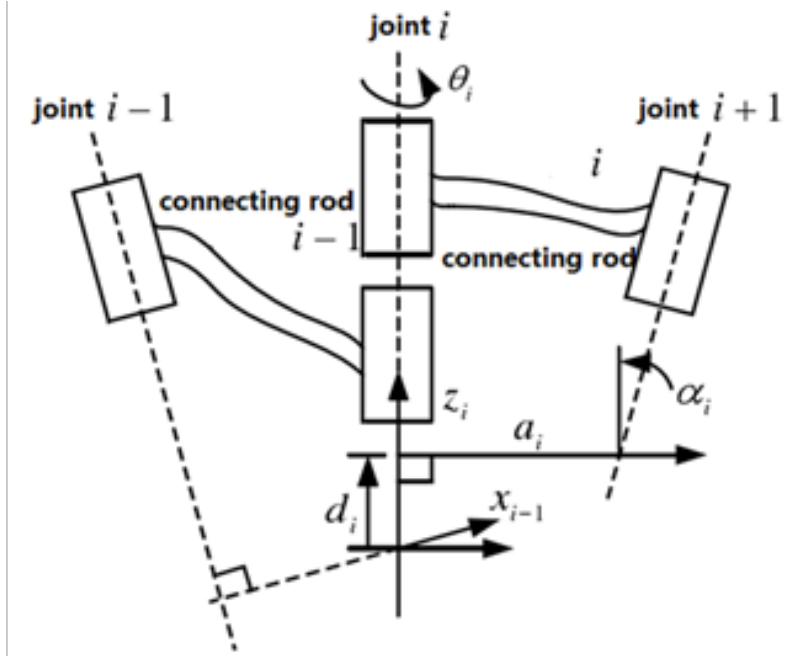

Fig. 4. Parameters of the $\mathrm{D}-\mathrm{H}$ of figure.

\section{Object identification and location}

Object recognition is realized on computer, object features are recognized by machine learning, and objects are located by image processing.

\subsection{Support vector machine}

Machine learning algorithms, it can be trained with a small number of samples. SVM sample space is established, information of different samples is extracted and mapped to the sample space, and classification is conducted by establishing segmentation boundary (hyperplane) for different samples[13].

Take 50 image samples for each object again to identify the object. The images were converted into binary images after processing, as the judgment basis of the support vector 
machine, the recognition test results were shown in table 2 . The overall recognition success rate was $98 \%$.

Table 2. Identify test results t.

\begin{tabular}{cccc}
\hline $\begin{array}{c}\text { Object } \\
\text { color }\end{array}$ & $\begin{array}{c}\text { Identificatio } \\
\text { Nnumber }\end{array}$ & $\begin{array}{c}\text { Number of } \\
\text { successful identificate }\end{array}$ & $\begin{array}{c}\text { Recognition } \\
\text { rate }\end{array}$ \\
\hline Red artifacts & 50 & 50 & $100 \%$ \\
Black artifacts & 50 & 49 & $98 \%$ \\
Spring artifacts & 50 & 49 & $98 \%$ \\
Blue artifacts & 50 & 50 & $100 \%$ \\
\hline
\end{tabular}

\subsection{Position of object}

After the classification of the object is determined, the appropriate grasping method is selected according to the shape of the object for grasping. The position of the object with circle characteristics is determined by detecting the position of the center of the circle. The direction of the object is arbitrary.Hough circle transform (HFT) is an accumulator based voting mechanism used to detect circular features. For a circle with a center of $(a, b)$ and a radius of $r$, the functional expression of the circle is shown in formula (3).

$$
(x-a)^{2}+(y-b)^{2}=r^{2}
$$

Taking the edge point as the center, calculate the point within the set maximum radius in both directions of the line. Calculate the distance from all edge points to the center of the circle and classify them according to the classification criteria. The radius of the circle is determined according to the linear density of the point, and the detection result is shown in figure 7.

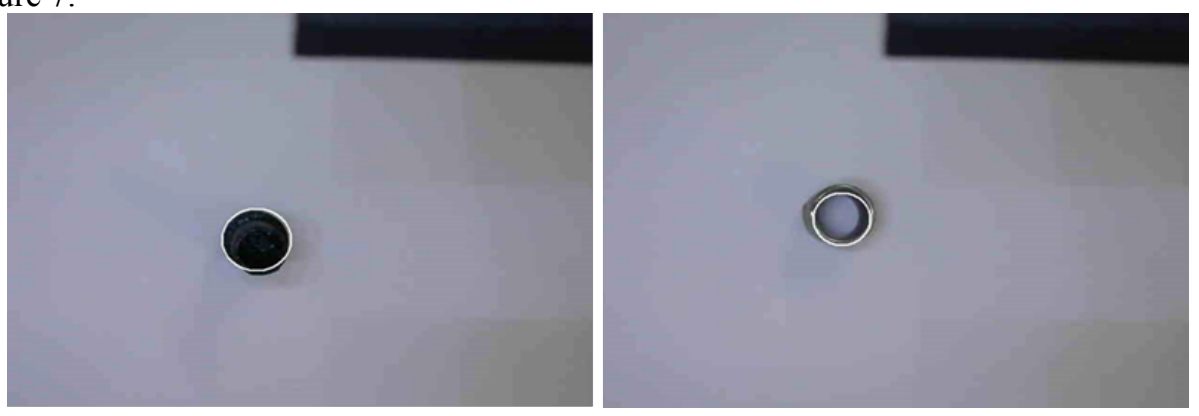

Fig. 7. Hough gradient method detects circular feature objects.

\section{Direct linear calibration}

The method to realize the direct linear calibration method is: place the workpiece on the conveyor belt, use the moving workpiece detection algorithm to detect the position of the workpiece, and read the coordinate information of the workpiece in the image space[14]. The industrial robot trainer is used to move the robot gripper on the manipulator to the position above the workpiece centroid and read the position coordinate information of the manipulator at this time. Read the position coordinates of the workpiece at different positions on the conveyor belt, establish the mapping relationship between the position coordinates of the workpiece in the image coordinate system $(\mathrm{U}, \mathrm{V})$ and the position coordinates of the mechanical arm coordinate system $\left(X_{w}, Y_{w}, Z_{w}\right)$, and complete the calibration of the camera. 
In this paper, 30 points in different positions were selected on the conveyor belt to place the workpiece, and the coordinates in $(\mathrm{U}, \mathrm{V})$ and $\left(X_{w}, Y_{w}, Z_{w}\right)$ of the workpiece in 30 positions were read, as shown in table 3.

Table 3. The position coordinates of the workpiece in the image coordinate system and the robot coordinate system.

\begin{tabular}{cccccc}
\hline $\begin{array}{c}\text { Serial } \\
\text { number }\end{array}$ & $\begin{array}{c}\text { Image } \\
\text { coordinates }\end{array}$ & $\begin{array}{c}\text { Robot } \\
\text { coordinates }\end{array}$ & $\begin{array}{c}\text { Serial } \\
\text { numbr }\end{array}$ & $\begin{array}{c}\text { Image } \\
\text { coordinates }\end{array}$ & $\begin{array}{c}\text { Robot } \\
\text { coordinates }\end{array}$ \\
\hline 1 & 679 & -215.53 & 16 & 555 & -44.55 \\
2 & 642 & -170 & 17 & 552 & -39.31 \\
3 & 640 & -167.86 & 18 & 549 & -33.58 \\
4 & 630 & -154.28 & 19 & 543 & -24.26 \\
5 & 622 & -145.44 & 20 & 540 & -19.8 \\
6 & 612 & -132.92 & 21 & 535 & -8.15 \\
7 & 604 & -121.85 & 22 & 531 & -0.06 \\
8 & 590 & -101.86 & 23 & 528 & 6.8 \\
9 & 583 & -91.57 & 24 & 526 & 11.94 \\
10 & 577 & -81.77 & 25 & 523 & 17.47 \\
11 & 572 & -75.46 & 26 & 520 & 13.3 \\
12 & 567 & -68.18 & 27 & 519 & 27.96 \\
13 & 564 & -63.9 & 28 & 516 & 33.39 \\
14 & 562 & -56.49 & 29 & 514 & 39.12 \\
15 & 559 & -51.83 & 30 & 512 & 43.97 \\
\hline
\end{tabular}

The coordinate data in table 3 is imported into MATLAB, and the coordinate relation curve of the workpiece in $(\mathrm{U}, \mathrm{V})$ and $\left(X_{w}, Y_{w}, Z_{w}\right)$ is drawn on MATLAB. The coordinate relation curve between the image coordinate system and the workpiece position in the robot coordinate system is shown in figure 8.

In order to obtain the functional relation expression of the curve in the figure 8 , the curve in figure 8 needs to be fitted and The curve after fitting is shown in figure 9. Because of the first-order linear function, there will be a large error and generated the robot arm cannot be guided to track and grasp accurately. In this paper, the second-order least square method is used to complete the fitting. The mathematical expression is formula (4), where $a, b$ and $c$ are the parameters obtained by curve fitting. When the image processing algorithm substitutes the centroid coordinates of the extracted moving workpiece into the function expression, the position coordinates of the workpiece can be calculated.

$$
f(x)=a * x^{2}+b * x+c
$$

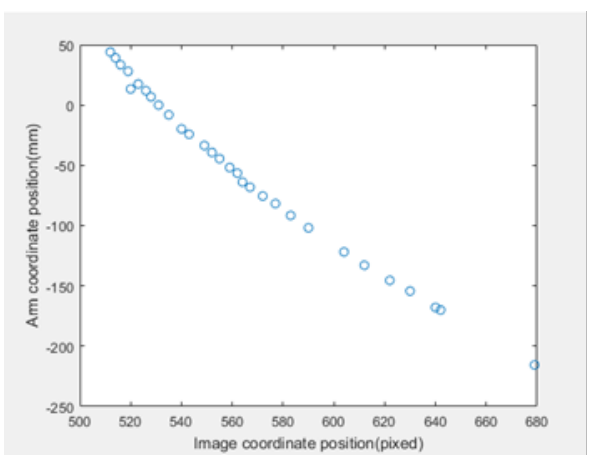

Fig. 8. The curve before fitting. 


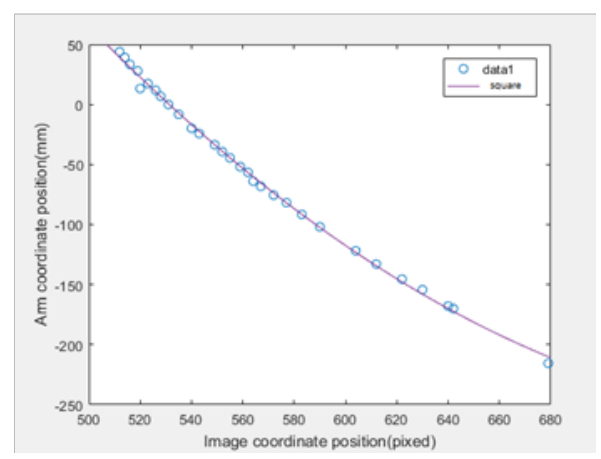

Fig. 9. The fitted curve.

\section{Conclusion}

After the experimental platform is set up and each coordinate is calibrated, static workpiece is placed for grasping. The image coordinate, the coordinate calculated by the fitting function and the actual captured coordinate are shown in table 4 . The actual captured artifact is shown in figure 10 and the grasping success rate of workpiece with different colors is shown in table 5. From the grasping results, the color characteristics of red and blue workpiece are obvious and the grasping success rate is high, while the color characteristics of black workpiece and spring workpiece are not obvious and the grasping success rate is slightly lower.The maximum detection error of uniaxial coordinate data of each workpiece is within $0.5 \mathrm{~mm}$, which meets the grasping requirements of mechanical arm.It is of reference significance for engineering applications. In the next step, on the basis of the research results, the robot arm will be further studied to dynamically grasp objects, so that the industrial robot can accurately grasp moving objects in space.

Table 4. Position data of camera inspection and robot teaching.

\begin{tabular}{ccc}
\hline $\begin{array}{c}\text { Serial } \\
\text { number }\end{array}$ & $\begin{array}{c}\text { data } \\
\text { name }\end{array}$ & $\begin{array}{c}\text { experimental } \\
\text { result }\end{array}$ \\
\hline \multirow{2}{*}{1} & Camera detection position $\mathrm{U}(\mathrm{x}, \mathrm{y})$ & $(678,235.27)$ \\
& Theoretical calculated position $\mathrm{f}(\mathrm{x}, \mathrm{y})$ & $(-190.35,96.5)$ \\
& Actual grab position $\mathrm{y}(\mathrm{x}, \mathrm{y})$ & $(575,190.20,96.5)$ \\
\hline \multirow{2}{*}{2} & Camera detection position $\mathrm{U}(\mathrm{x}, \mathrm{y})$ & $(-78.36,108.35)$ \\
& Theoretical calculated position $\mathrm{f}(\mathrm{x}, \mathrm{y})$ & $(-78.86,108.35)$ \\
\hline \multirow{3}{*}{3} & Actual grab position $\mathrm{y}(\mathrm{x}, \mathrm{y})$ & $(518,105.56)$ \\
& Camera detection position $\mathrm{U}(\mathrm{x}, \mathrm{y})$ & $(30.53,95.25)$ \\
& Theoretical calculated position $\mathrm{f}(\mathrm{x}, \mathrm{y})$ & $(30.56,95.25)$ \\
\hline
\end{tabular}

Table 5. Experimental grab success rate.

\begin{tabular}{cccc}
\hline Object color & Grab the number & Number of successful attempts & Recognition rate \\
\hline Red artifacts & 50 & 50 & $100 \%$ \\
Black artifacts & 50 & 49 & $98 \%$ \\
Spring artifacts & 50 & 48 & $96 \%$ \\
Blue artifacts & 50 & 50 & $100 \%$ \\
\hline
\end{tabular}



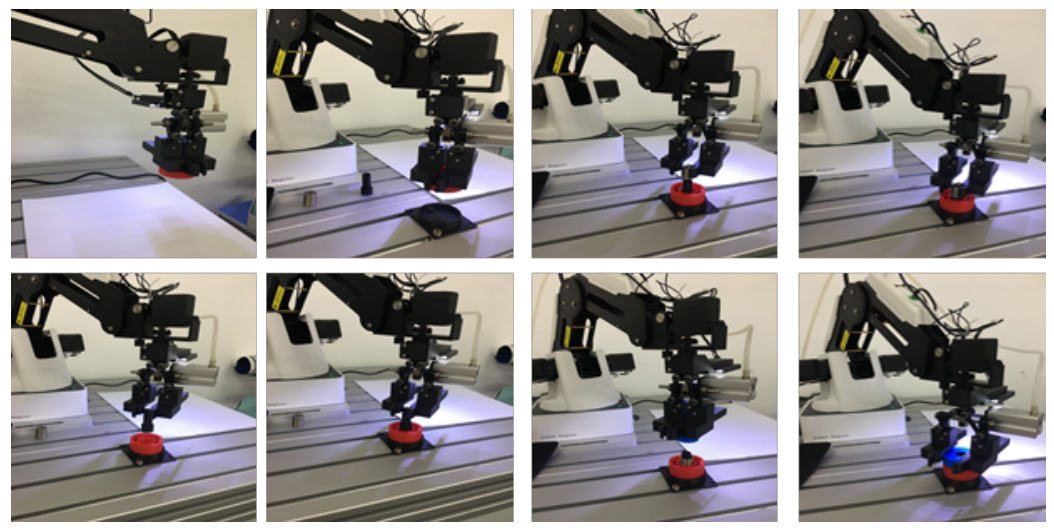

Fig. 10. The actual capture and placement of the workpiece.

\section{References}

1. Zhang De-hong, Dai Yan-xia. Construction of Robot Vision Handling System and Software Development[J]. Packaging Engineering, 2019, 40(1): 149 -155.

2. Zhai Jing-mei, Dong Peng-fei, Zhang Tie. Positioning and Grasping System Design of Industrial Robot Based on Visual Guidance[J]. Manufacturing Automation, 2014. 30(5): 45- 49.

3. Zhai Jing-mei, Dong Peng-fei, Zhang Tie. Positioning anđ Grasping System Design of Industrial Robot Based on Visual Guidance[J]. Manufacturing Automation, 2014. 30(5): 45- 49.

4. Wang Yao-nan, Chen Tie-jian, He Zhen-dong, et al. Review on the Machine Vision Measurement anđContro1 Technology for Intelligent Manufacturing [J]. Control Technology and Applications, 2015, 32(3):273 -286.

5. Liu Zheng-qiong, WAN Peng. Recognition and Grappling System for Workpieces Exceeđing the Visual Field Based on Machine Vision [J]. Robot, 2018, 40(3):294 308.

6. Zheng Hua, CHEN Jun. JIN Hong-zhang. Research on Kinematics and Visual Grapping SCARA Robot [J]. Modular Machine Tool \& Automatic Manufacturing Technique, 2017(6): 50 -58.

7. Chen Zhi-wei, XU Shi-xu, LIU Yun-peng, et al. Design of Paralle1 Robot Plane Grasping System Basedon Visual Screening[J]. Manufacturing Automation,2018, 40(5): 4447.

8. Hill J. Real Time Control of a Robot with a Mobile Camera[J]. Proc.int. symp. on Industrial Robots, 1979.

9. E. Malis, F. Chaumette, and S. Boudet, "2-1/2D visual servoing,"IEEE Trans. Robot. Automat,vol. 15, pp. 238-250, Apr. 1999.

10. Milan Sonka, Vaclav Hlavac, Roger Boyle. Image processing, analysis and machine vision [M]. BeiJing: Tsinghua University Press, 2016:232-261.

11. Ryberg A, Lennartson B, Christiansson A K, et al. Analysis and evaluation of a general cameramodel[J]. Computer Vision \& Image Understanding, 2011, 115(11):1503-1515.

12. Zhou Zhi-hua. machine learning [M].BeiJing: Tsinghua University Press,2016:121-140. 
13. Zhang Z. A Flexible New Technique for Camera Calibration[C]// IEEE Transactions on Pattern Analysis and Machine Intelligence. 2000:1330 -1334.

14. FREEMAN W T, JONEST R, PASZTOR E C. Example-based super-resolution[J]. IEEE Computer Graphics and Applications, 2002,22(2) :56 - 65. 